\title{
Low Density Parity Check Coding in OFDM-PON Systems
}

\author{
Chunlei Zhang ${ }^{1, *}$, Xiao Xiao ${ }^{2}$, Kai Zhao ${ }^{1}$ and Xunfa Yang ${ }^{1}$ \\ ${ }^{1}$ School of Electronic and Information Engineering, Lanzhou Jiaotong University, Lanzhou, China \\ ${ }^{2}$ Chengdu Metro Co. Ltd, Chengdu, China \\ ${ }^{*}$ Corresponding author
}

\begin{abstract}
To meet the demands of different high bandwidth service, OFDM-PON has been considered as one of the candidates for NG-PON2. In this paper, downstream transmission performance based on LDPC-OFDM coding PON system is studied. The simulation results show that employing the LDPC-OFDM coding in PON system can achieve $10 \mathrm{~Gb} / \mathrm{s}$ downstream transmission. The transmission performance of the LDPC-OFDM system is improved $4.2 \mathrm{~dB}$ than uncoded system at the BER of $10^{-4}$. When the SNR is greater than 2. $6 \mathrm{~dB}$, the Gallager method has better BER performance than the Mackay and BIBD construction schemes, and when the SNR is greater than 4. $6 \mathrm{~dB}$, LLR-BP and the improved decoding algorithm enables to improve system performance.
\end{abstract}

Keywords-low-density parity-check (LDPC); passive optical network (PON); orthogonal frequency division multiplexing (OFDM)

\section{INTRODUCTION}

With the development of high-bandwidth services, such as IPTV and video-on-demand, broadband access network has been regarded as a promising technology in order to meet access bandwidth requirement. OFDM-PON has been researched as a promising candidate for NG-PON2, due to its benefits such as strong dispersion tolerance, high spectral efficiency, flexible resource allocation and potentially low cost [1-4]. In a typical PON system, the traffic is transmitted from a user to others via OLT, and suffered from inevitable O-E-O conversion with electrical processes.

LDPC codes, proposed by Gallager in 1962, are linear block codes based on sparse parity check matrix. LDPC codes use random coding method, and decoding uses BP (Belief Propagation) algorithm based on iterative thought in Tanner graph. Since rediscovered in 1996, LDPC code has taken up the leader position of current scenario of forward error correction because of its Shannon-limit-approaching performance and parallel decoding structure [5-7]. Practical LDPC coding schemes have been designed for fixed and mobile broadband wireless access systems (IEEE802.16d/e). Recently, some researchers turn their eyes to its applications to optical communications [8-11].

In this paper, the LDPC-OFDM coded modulation in the passive optical networks has been studied. The paper is organized as follows. In section II, the system architecture of WDM-OFDM-PON with LDPC code is given. The performance of the proposed system is simulated in section III.
The transmission performance comparison of different coding schemes and decoding algorithms are given. At last, we conclude the paper.

\section{LDPC-OFDM PON SYSTEM}

\section{A. LDPC Code}

Low Density Parity Check (LDPC) coding not only has high coding gain, but also has large degree of freedom in the design of the coding structure [6]. It can map an information sequence into the codeword sequence by a linear generator matrix $\boldsymbol{G}$. The generator matrix $\boldsymbol{G}$ may be uniquely defined by a parity check matrix $\boldsymbol{H}$. By Gaussian elimination method, a dimension of $m \times n$ matrix $\boldsymbol{H}$ may be transformed into the form of Equation 1.

$$
H=\left[\begin{array}{ll}
I_{(n-k) \times(n-k)} & P_{(n-k) \times k}^{T} \\
0 & 0
\end{array}\right]
$$

If $H$ is full rank, there is no all-zero row after the change. Then $m=n-k$, and the bit rate is $r=\frac{n-m}{n}=1-\frac{m}{n}$. But sometimes rows of the matrix $\mathrm{H}$ does not have linear independence, then $m>n-k$, that is the bit rate $r>1-\frac{m}{n}$. From the above conversion, the generator matrix in the system form can be obtained $G=\left[\begin{array}{ll}P_{k \times(n-k)}^{T} & I_{k \times k}\end{array}\right]$, the information sequence $u=\left[u_{1}, u_{2}, \cdots, u_{k}\right]$ maps into codeword by $c=u \cdot G$. At the same time the corresponding Tanner graph needs to avoid short loops. The LDPC minimum distance based on the Tanner limits is:

$$
d \geq\left\{\begin{array}{c}
1+\frac{w_{c}}{w_{c}-2}\left(\left(w_{c}-1\right)^{\left\lfloor\frac{g-2}{4}\right\rfloor}-1\right), \frac{g}{2}=2 m+1 \\
1+\frac{w_{c}}{w_{c}-2}\left(\left(w_{c}-1\right)^{\left\lfloor\frac{\underline{-}-2}{4}\right\rfloor}-1\right)+\left(w_{c}-1\right)^{\left\lfloor\frac{g-2}{4}\right\rfloor}, \frac{g}{2}=2 m
\end{array}\right.
$$

In the Equation 2, $g$ represents the perimeter; $w_{c}$ represents the column weight; $d$ represents the minimum coding distance. How to reduce the relatively high latency and algorithmic complexity of the LDPC coding become an urgent problem. 


\section{B. OFDM Modulation}

Figure I illustrates the transmitter and receiver of the OFDM-PON system. Binary input data is converted from a high-speed serial signal to a relative low-speed parallel signal firstly and encoded using identical LDPC codes. After using QAM to map bit stream data onto each OFDM subcarrier, the modulated subcarriers are then presented as an input of the inverse fast Fourier transform (IFFT). The parallel output signal of IFFT is converted back to a serial signal (P/S). After $\mathrm{P} / \mathrm{S}$, a cyclic prefix (CP) is added to make the system resilient to fiber dispersion. Next, the discrete signal is passed through a process of digital-to-analog (D/A) conversion. The baseband OFDM signal is mixed with a local optical oscillator and changed to an optical RF OFDM signal. At the receiver, the optical OFDM signal is converted to a baseband OFDM electrical signal after detection by a photodiode. After analogto-digital (A/D) conversion, the CP of the OFDM signal is removed and next series signals convert to parallel signals (S/P). The OFDM signal is then de-multiplexed by the fast Fourier transformation (FFT). Then the symbols are constellation demapped and at last the output data are got after parallel signals convert to series signals.

\section{System Architecture}

The proposed LDPC-OFDM PON system is illustrated in Figure II. The optical OFDM system includes coherent optical OFDM (CO-OFDM) and direct detection optical OFDM (DDO-OFDM) [4, 9-11]. We use direct detection system to receive in our system considering the transmission distance and the system costs. The optical line terminal (OLT) consists of LDPC encode, OFDM transmitter and receiver, upconverter and downconverter, E/O and O/E converter.

In the transmitter, data are encoded in downstream LDPC encode module, the encoding process carried out in the computer, and then the data are sent to the downlink OFDM transmitter module. In this module data are modulated into OFDM data, the modulated data are converted to downlink RF OFDM signals by the up-converter, and finally the signals go through the optical modulator to achieve electrical to optical conversion (E/O), generating downstream optical OFDM signal. The downstream optical signal passes through an optical circular (OC) to send to the fiber channel downlink optical link transmitted to ONUs. Optical signal is divided into a plurality of beams through a splitter, and finally sent to each ONU.

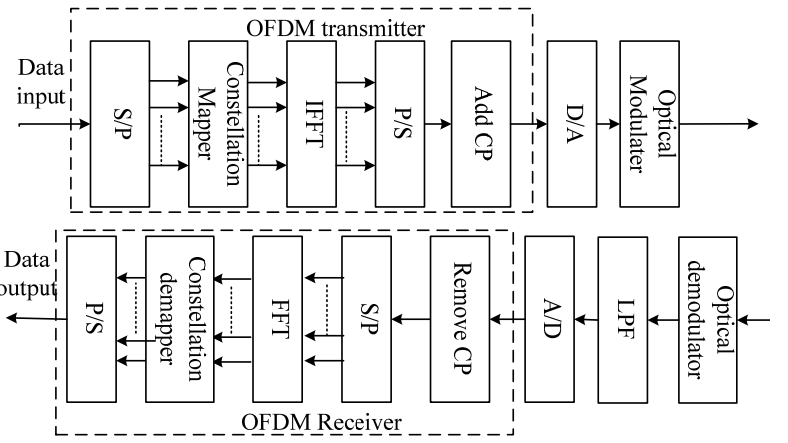

FIGURE I. PRINCIPLE DIAGRAM OF THE OFDM-PON TRANSMITTER AND RECEIVER

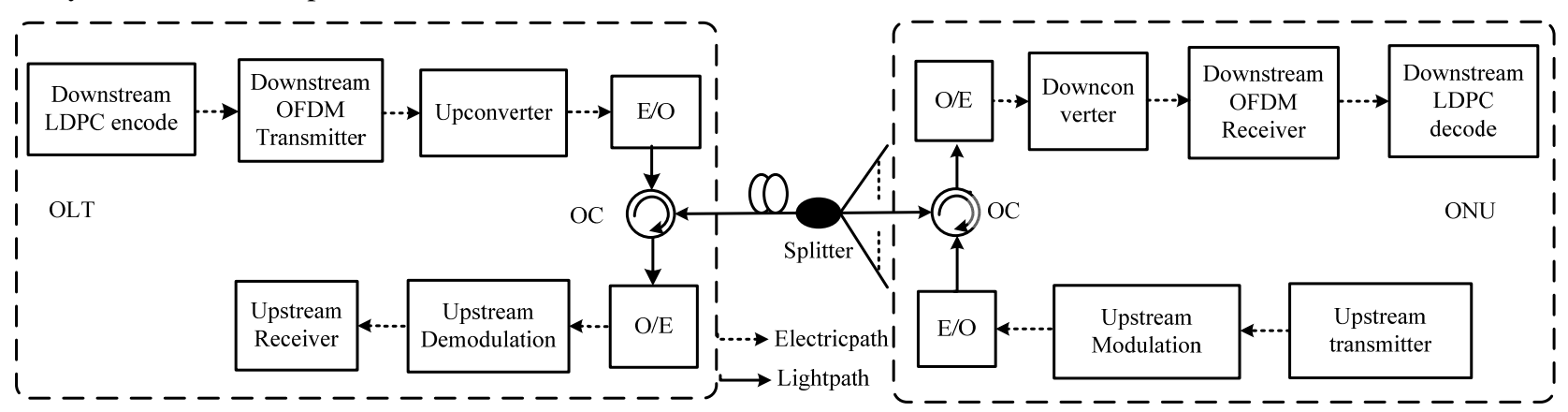

FIGURE II. PRINCIPLE DIAGRAM OF LDPC-OFDM PON SYSTEM

\section{SimULATION RESUlTS}

The LDPC-coded OFDM signals are produced through offline MATLAB programming. The LDPC $(5120,4096)$ code of girt 6 , a code rate of 0.8 is used. OFDM signals map using 4 QAM. The RF carrier frequency is $10 \mathrm{GHz}$, the number of sub-carriers is 512 , oversampling factor is 2 . And then by the arbitrary waveform generator generates code modulation data of $10 \mathrm{~Gb} / \mathrm{s}$, using optical intensity modulator (IM) to externally modulate DFB-LD for downlink transmission. The number of OLT downlink carriers is 8, with frequency distribution from 193.1 to $193.8 \mathrm{THz}$. The downlink coded modulated optical signals go to optical network units (ONUs) through the optical distribution network (ODN). Wherein, ODN consists with a $20 \mathrm{~km}$ single-mode fiber (SMF) and a splitter, the ONUs number is eight. In the ONU, the downlink signal is sampled from real-time oscilloscope after $\mathrm{O} / \mathrm{E}$ conversion, and finally gets the downstream data after demodulation and decoding.

The BER curves with and without LDPC coding and back to back without coding are explored in Figure 3. It shows that LDPC-coded OFDM signals downlink transmission at a total channel BER of $10^{-3}$ the OSNR is $5.1 \mathrm{~dB}$ for $20 \mathrm{~km}$, and the OSNR is $8.6 \mathrm{~dB}$ without coding. That means there exists a $3.5 \mathrm{~dB}$ improvement between two schemes. Compared with back-to-back transmission without LDPC coding the OSNR is $7.4 \mathrm{~dB}$, there exists $2.3 \mathrm{~dB}$ improvement. Simulation results are shown in Figure III. 


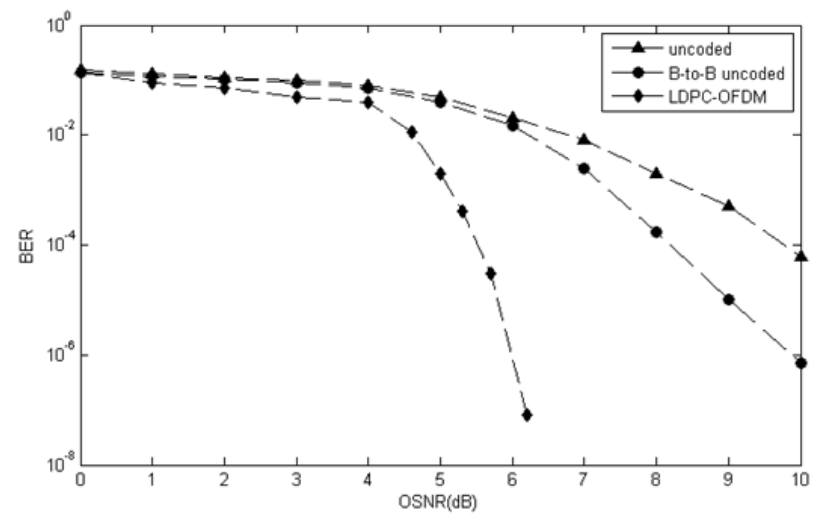

FIGURE III. SYSTEM BER PERFORMANCE OF LDPC-OFDM PON

We compare the BER performance of different code construction schemes and the results are given in Figure IV These LDPC code construction schemes are Gallager, Mackay and BIBD construction schemes. The results show that when SNR is less than $2.6 \mathrm{~dB}$, Mackay and BIBD construction methods have better BER performance; when the SNR is greater than $2.6 \mathrm{~dB}$, the $\mathrm{BER}$ performance of Gallager construction method is better; when the SNR is $5 \mathrm{~dB}$ the BER reaches $10^{-3}$.

The BER performance with different decoding algorithms show as Figure V. We explored LLR-BP, improved LLR-BP and bit-flipping (BF) three kinds of decoding algorithms. The results show that when SNR is less than $4.6 \mathrm{~dB}$, the BER performance of the BP scheme is the best; and when the SNR is greater than $4.6 \mathrm{~dB}$, the performance of LLR-BP and improved LLR-BP schemes is better. The improved LLR-BP scheme reduces the complexity and has better performance in high SNR.

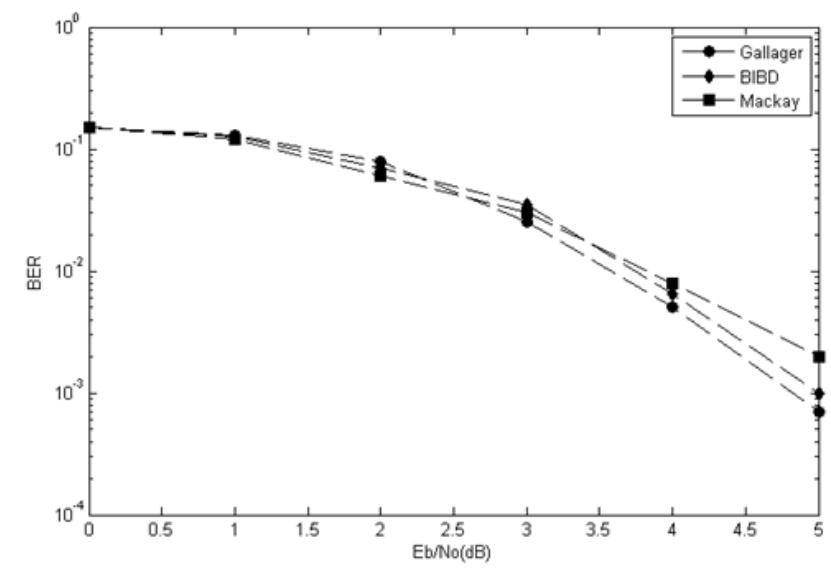

FIGURE IV. BER PERFORMANCE WITH DIFFERENT CODING SCHEMES

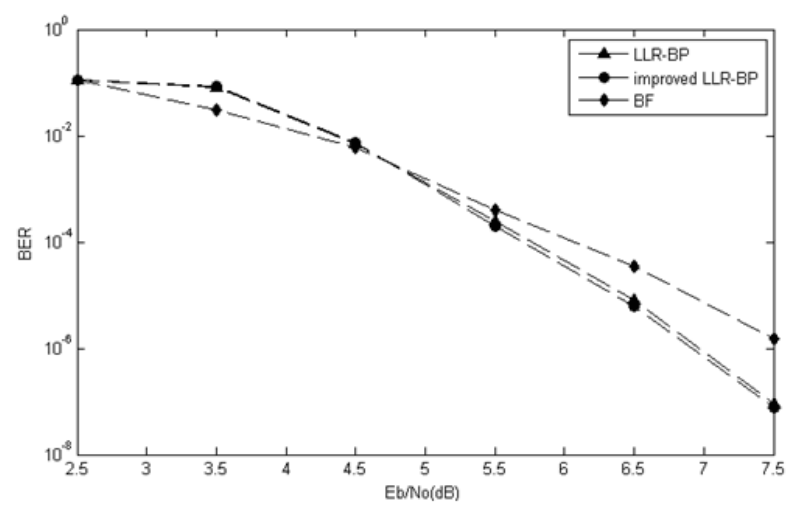

FIGURE V. BER PERFORMANCE WITH DIFFERENT DECODING ALGORITHMS

\section{CONCLUSION}

We have proposed the LDPC-OFDM coded modulation in a passive optical network. Through theoretical analysis and simulation results show that by the LDPC-OFDM coded modulation signals in a passive optical network can achieve 10 $\mathrm{Gb} / \mathrm{s}$ downstream transmission over $20 \mathrm{~km}$ single-mode fiber. When the BER is $10^{-4}$, the system transmission performance of LDPC-OFDM coding has a $4.2 \mathrm{~dB}$ improvement than uncoded system. When the SNR is greater than $2.6 \mathrm{~dB}$, Gallager construction method represents better BER performance than Mackay and BIBD construction schemes, and when the SNR is greater than $4.6 \mathrm{~dB}$, LLR-BP and the improved decoding algorithm enables to improve system performance.

\section{ACKNOWLEDGMENT}

This work is partly supported by Natural Science Funds of Gansu Province under Contract No.148RJZA047. First Author is corresponding author.

\section{REFERENCES}

[1] D. Nesset, "NG-PON2 technology and standards,” Journal of Lightwave Technology, vol. 33, no. 5, pp. 1136-1143, Mar. 2015

[2] C. Chen, C. F. Zhang, Y. Feng, and K. Qiu, "Bidirectional radio frequency up-converted orthogonal frequency-division multiple access passive optical network with novel source-free optical network units using four-wave mixing in semiconductor optical amplifier," IEEE Photonics Technology Letters, vol. 24, no. 4, pp. 2206-2209, 2012.

[3] A. Tsokanos, E. Giacoumidis, G. Zardas, A. Kavatzikidis, N.P. Diamantopoulos, I. Aldaya, I. Tomkos, "Reductions of peak-to-average power ratio and optical beat interference in cost-effective OFDMAPONs,” Photonic Network Communications, vol. 26, no. 2, pp. 44-52, 2013.

[4] Yuang Maria C, Polung Tien, Darzu Hsu, Chen Shingyu, Wei Chiachien, Shih Julin, Chen Jyehong, "A high performance OFDMA-PON system architecture and medium access control," Journal of Lightwave Technology, vol. 30, no. 11, pp. 1685-1693, 2012.

[5] Jian-guo Yuan, Ya Xie, Lin Wang, Sheng Huang and Yong Wang, “A novel concatenated code based on the improved SCG-LDPC code for optical transmission system,” Optoelectronics Letters, vol. 9, no.1, pp. 42-44, 2013.

[6] Billini S, Ferrari M, Tomasoni A, Costantini C, Razzette L, Gavioli G, "LDPC design for block differential modulation in optical communications," Journal of Lightwave Technology, vol. 33, no. 1, pp.78-88, 2015. 
[7] Yang Q and He Z, "1Tb/s large girth LDPC-coded coherent optical OFDM transmission over 1040km standard single-mode fiber,” Optical Fiber Communication Conference and Exposition and the National Fiber Optic Engineers Conference, OFC/NFOEC 2011.

[8] Wang Y. and J. Cao, "Performance analysis of atmospheric laser communication system basing on asymmetrically clipped optical orthogonal frequency division multiplexing intensity modulation and lower density parity check code,” Zhongguo Jiguang/Chinese Journal of Lasers, vol. 37, no. 12, pp.3031-3036, 2010

[9] X. Jiang, "Progress and Challenges for high speed optical access networks," $20^{\text {th }}$ Annual Wireless and Optical Communications Conference (WOCC), 2011

[10] Cao Zizheng, Yu Jianjun, Zhou Hui, Wang Wenpei, Xia Minmin, Wang Jing, et al., "WDM-ROF-PON architecture for flexible wireless and wire-line layout," Optical Communications and Networking, vol. 2, no. 2, pp.117-121, 2010.

[11] Yu Jianjun, Jia Zhensheng, Wang Ting, Chang Gee-Kung, Ellinas Georgios, "Demonstration of a novel WDM-PON access network compatible with RoF system to provide $2.5 \mathrm{~Gb} / \mathrm{s}$ per channel symmetric data services," Proc. Optical Fiber Communication Conference and Exposition and the National Fiber Optic Engineers Conference OFC2007, Paper OThM5. 